\title{
RADIOGAPS: STABILITY OF ELECTRON/POSITRON-JETS IN LARGE-SCALE MAGNETIC FIELDS
}

\author{
U. Achatz', H. Lesch ${ }^{2}$, R. Schlickeiser ${ }^{1}$ \\ ${ }_{1}^{1}$ Max-Planck-Institut für Radioastronomie. Auf dem Hügel 69, 5300 \\ Bonn 1, F.R.G. \\ ${ }^{2}$ Landessternwarte Königstuhl. 6900 Heidelberg 1. F.R.G.
}

\begin{abstract}
Radio gaps, i.e. regions of strongly reduced radio ( and also optical ) intensity between the galactic nucleus ( AGN) and the radio jets in active radio galaxies, are a rather prominent feature of many of these sources. With the aim to explain this phenomenon we consider the evolution of the relativistic electron spectrum in such a source with the relevant plasma kinetic equations. We consider a possible acceleration process for relativistic electrons and positrons near the accreting central object which is based on the continuous interplay of stochastic Fermi acceleration processes and radiation losses of energetic particles. The resulting distribution function heats the background medium efficiently through the oblique Langmuir synchrotron instability once the threshold plasma energy density for the modulation instability is exceeded resulting in the outflow of material. By the concerted action of synchrotron losses and adiabatic focussing in the ordered, spatially decaying magnetic field $B_{0}$ the isotropic relativistic electron distribution function rapidly develops into two oppositely directed beam distributions, explaining the formation of compact, superluminal radio jets near the $A G N$ and the radio gap phenomenon. The stability of the electron/positron beam in the magnetized background plasma is studied by treating it as a cold beam. The linear growth rates for parallel propagating longitudinal and transversal waves are calculated. Whereas the former give rise to heating of the background plasma, the low-frequency transversal (Alfven-) waves scatter the beam to an isotropic distribution function which then reveals itself again by synchrotron radiation. The mean free path for this isotropization process is calculated and compared with the length of the radio gaps. A discussion of the results is given in Achatz et al. ( 1989 ).
\end{abstract}

References:

Achatz, U.. Lesch, H., Schlickeiser, R. ( 1989 ) 'Jets and hot spots in extragalactic radio sources'. Astron. Astrophys., in prep. 